\title{
Data Ethics Club: creating a collaborative space to discuss data ethics
}

\author{
Nina H. Di Cara* ${ }^{1}$, Natalie Zelenka*2 ${ }^{*}$, Huw Day ${ }^{3}$, Euan D. S. Bennet ${ }^{4}$, Vanessa \\ Hanschke ${ }^{5}$, Valerio Maggio ${ }^{1}$, Ola Michalec ${ }^{5,6}$, Charles Radclyffe ${ }^{5}$, Roman

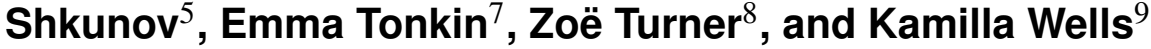 \\ ${ }^{1}$ MRC Integrative Epidemiology Unit, University of Bristol, UK \\ ${ }^{2}$ Jean Golding Institute for Data Intensive Research, University of Bristol, UK \\ ${ }^{3}$ School of Mathematics, University of Bristol, UK \\ ${ }^{4}$ Bristol Veterinary School, University of Bristol, UK \\ ${ }^{5}$ Department of Computer Science, University of Bristol, UK \\ ${ }^{6}$ Bristol Cyber Security Group, University of Bristol, UK \\ ${ }^{7}$ Digital Health, University of Bristol, UK \\ ${ }^{8}$ Nottinghamshire Healthcare NHS Foundation Trust, UK \\ ${ }^{9}$ Australian Public Service, Brisbane, Australia \\ *Joint first authors
}

\begin{abstract}
Awareness and management of ethical issues in data science is becoming increasingly relevant to us all, and a crucial skill for data scientists. Discussion of contemporary issues in collaborative and interdisciplinary spaces is an engaging way to allow data science work to be influenced by those with expertise in philosophy, history, sociology and beyond, and so improve the ability of data scientists to think critically about the ethics of their work. However, opportunities to do so are limited. Data Ethics Club (based at dataethicsclub.com) is a fortnightly discussion group about data science and ethics, whose community-generated resources are hosted in an open online repository. This repository includes a list of data science and ethics materials around multiple topics of interest, alongside processes and templates for leading an online data ethics discussion group. These meetings and materials are designed to reduce the barrier to learning, reflection and critique on data science and ethics for all interested parties, with the broader aim of building ethics into the cultural fabric of quality data science work.
\end{abstract}

Keywords: data ethics, responsible innovation, journal club, open science, interdisciplinary

\section{THE BIGGER PICTURE}

The Data Ethics Club aims to help data scientists, analysts, and other interested parties up-skill in their knowledge of data ethics through discussions in an interdisciplinary setting that encourages individuals to explore their own ethical challenges and boundaries. Our aim is to make conversations about data ethics more common in data science spaces, and for these conversations to be informed by the critical work taking place in sociological and philosophical disciplines. Our longer term ambitions are for a diverse community to continue to develop the resources we have created, and for more groups in in other locations and time zones to be established. By bringing data ethics to people in a range of roles and organisations we can increase the awareness of ethics in our day-to-day settings, and work towards preventing future injustices being aided and abetted by data science.

\section{INTRODUCTION}

Data science and digital technologies have a huge role in all of our day to day lives, which is increasing year on year. There are countless examples of how data science shapes and influences our daily routines: from vehicles employing satellite navigation technologies [1], through emotional recognition tools applied to the monitoring of school children's well-being [2] to automated translation and captioning widely used in the entertainment industry [3]. While these technologies are incredibly useful and represent exciting advances, the extent of their reach in our lives leaves those developing these technologies with a huge amount of collective power over the way the world is modelled and interpreted using data. It has taken a series of public scandals, and the voices of key groups, whistle-blowers, and researchers such as the Algorithmic Justice League [4], Christopher Wylie and Brittany Kaiser ${ }^{1}$, Timnit Gebru and Margaret Mitchell ${ }^{2}$ for us to take stock of the role of data scientists as the architects of digital systems. We are

\footnotetext{
${ }^{1}$ Whistle-blowers from Cambridge Analytica

${ }^{2}$ Researchers from Google's Ethical AI team who spoke out after being dismissed
} 
beginning to better understand that data scientists can influence who shapes, accesses and ultimately benefits from the adoption of digital innovations. Whilst this growing number of concerns about the unfair impacts of new data-centric technologies have highlighted the importance of including diverse voices when building data science tools, every year still brings new examples of technologies doing harm to the people who interact with them.

So, why do we continue to face scandals that leave us asking "why did no one ask the right questions about this earlier?". The end products/services of data science are often subject to the decisions of multiple stakeholders, at multiple levels of organisations. Yet, ethical approval is not typically part of the life-cycle of a data science project. Where it is, ethical review boards are primarily tasked with protecting participants and ensuring that data science research is conducted within the bounds of the law. When a project does not reach the threshold for institutional ethical review, there are few incentives to consider ethics. However, whilst data scientists do not generally embed critical work on data ethics, this does not mean it does not exist. There are whole fields of philosophical and sociological thought which contemplate, discuss and provide frameworks around questions that data scientists inadvertently encounter. For instance, is there a universal definition of fairness that we can apply in algorithmic systems [5]? What should we do with ill-gotten data if it can be used for the public good [6]? Can automated systems be used as 'neutral' tools in welfare services [7]? There is a vast landscape of knowledge and expertise on how to consider these questions in the context of different scenarios and points in the data science life cycle, from data cleaning to algorithmic decision making. However, engaging with this literature requires a disciplinary leap that data scientists are often not trained for, and there are rarely reciprocal opportunities for experts in philosophy or sociology to influence the day-to-day practice of data scientists.

Building interdisciplinary spaces where people from different backgrounds can come together to discuss the application of ethics to data science is one way in which we can begin to encourage data scientists to engage with critical material from other fields [8]. These spaces, which may be referred to as 'trading zones' [9] or 'communities of practice' [10], allow important lessons and concepts learned in the field of data ethics to reach those who are working in applied settings. They can also encourage consideration of ethical issues as part of the process of doing data science, and being a responsible data scientist [11]. This does require a willingness on the part of data scientists to engage with critical questions about ethics of their work that goes beyond data representativeness and fairness as problems to be solved. Ideally data scientists should have an understanding of the roles of power and influence in their work and how it affects others [12]. Data Ethics Club exists to create and facilitate spaces that address these needs, and to help build ethics into the cultural fabric of quality data science work.

\section{What is Data Ethics Club?}

Data Ethics Club is a fortnightly reading and discussion group held virtually, and currently hosted by University of Bristol staff and students (Figure 1). It is free to attend and open to everyone.

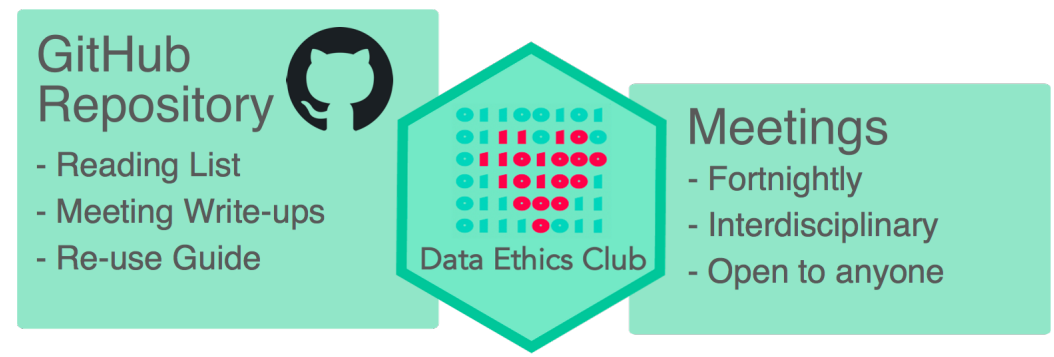

Figure 1. A visual overview of Data Ethics Club.

We have developed an open source repository ${ }^{3}$ of reusable resources that have been collaboratively developed over time to host an interdisciplinary data ethics discussion group. This includes our reading list, organisational materials and written records of our discussions. The repository encourages collaboration from new contributors who would like to add to the reading list, help to develop the reusable materials, or organise their own Data Ethics Club. Our meetings are open to anyone who would like to join, and we also hope that our resources will support other people who wish to run their own group.

The incentive for creating Data Ethics Club grew from a desire for a space that allows discussion of the intractable dilemmas that inevitably arise from thinking about ethical responsibility of data scientists in our complex world. Similarly to a traditional journal club [13, 14], Data Ethics Club is intended to be a place where anyone can come to learn about data ethics, and to share their expertise. However, unlike typical journal clubs it explicitly invites a wide range of attendees, from those who are professional ethicists and philosophers to those who might describe themselves

${ }^{3}$ https://github.com/very-good-science/data-ethics-club 
as analysts, statisticians, data managers, software developers, or simply interested citizens. Data Ethics Club also fulfils a need beyond being a space to learn about ethics. The group setting is an acknowledgement that ethics is something that is fundamentally co-constructed and relative; one person with one perspective simply cannot have all the answers [12, 15]. As such, participation in a discussion group becomes an awareness-raising exercise, where we can feel out the boundaries of what is acceptable to who, and why. Most importantly, we can start to understand what is important to ourselves, and what influence that may have on our own work.

Specifically, Data Ethics Club meetings aim to:

- Provide structured time and space for engaging with material about data ethics.

- Provide a collaborative environment where attendees can ask critical questions about the material, and engage in discussion with others so that each of us can challenge our own assumptions.

- Bring together a diverse group of people from across the data science community, including business owners, students, academics, industry-based data scientists, philosophers, policy makers, publishers and more.

Data Ethics Club is organised around a central repository where we share our outputs, reading list, collaborative work and organising materials. This repository is the subject of this article.

\section{OUTPUTS}

The Data Ethics Club repository is hosted on GitHub ${ }^{3}$ and has been in ongoing development since January 2021 with collaboration from approximately 40 different people across academia, government services, and private industry. Their contributions have been towards the three primary outputs of the repository:

- A reading list of discussion materials around the theme of data ethics.

- A repository structure which can be copied for anyone to start their own group, that contains organisational materials and an integrated website.

- Written outputs from meetings of the Data Ethics Club, co-produced by the attendees of the meeting.

These three outputs can all be found in the 'sites̀ub-folder of the repository, and are organised in a structure that prioritises the layout of the content on the associated website. The project remains live, and continues to develop and grow as time goes on, however a static release with a Digital Object Identifier made at the time of writing is available (doi:10.5281/zenodo.6243804). The repository is licensed with a CC-BY-4.0 license [16], roughly meaning that it can be re-used and re-mixed with accreditation. We describe the main outputs of the repository in more detail below.

\section{Reading list}

The 'reading' list for Data Ethics Club has been put together collaboratively by anyone interested in the topic. The material has been divided into sections to allow anyone to design a programme of reading based on their interests or the starting point of their group. It can also expand in the future with the addition of new resources and sub-sections as needed. At the point of writing the reading list is separated into the eleven subsections briefly described in Table 1. These sections have developed organically in order to organise the volume of content in the list into thematically similar groups. These groups give an idea of the Data Ethics Club community's broad definition of data ethics. We aim to understand not just the academic field of data ethics as it stands currently, but also where our established norms came from (e.g. in History of data science) as well as current examples of implementations of data ethics (e.g. Ethics in action and Data ethics in the public and private sectors). We also consider research culture to be a crucial element of ethical data science, and aim to include content that requires us to look at ourselves as a field and consider structural inequalities in our individual settings.

The reading list content varies in format, including journal articles, government reports, book chapters, YouTube videos and podcasts. Audio, video, long-form (like book chapters), very short-form (like poems), or closed access content are indicated in the list using an emoji key to help people find what they need.

\section{Online journal club repository materials}

The second output of the Data Ethics Club is the guides and materials for starting and running another group, specifically as an online journal club. We have created a 'How To' sub-section of the repository which contains all of our resources ready for re-use. This includes instructions on necessary tasks before the meeting, the structure and timing of the meeting itself, and the software we use to help facilitate the group.

The repository is hosted on GitHub and integrated with several useful features which could be re-used by forking or downloading the materials for those who do not use GitHub. Firstly, there is a custom issue template, designed for suggesting new reading materials, which invites the suggester to contribute three questions to be used as discussion 


\begin{tabular}{ll}
\hline Section & Description \\
\hline What is data ethics? & $\begin{array}{l}\text { Pieces that map the boundaries of data ethics, and introduce the concept } \\
\text { at a broad level. }\end{array}$ \\
The nature of data & $\begin{array}{l}\text { Exploring how we understand the data we use as a tool embedded with } \\
\text { assumptions rather than a static, objective object. } \\
\text { Moral philosophy for data science }\end{array}$ \\
History of data science & $\begin{array}{l}\text { The origins of data science and consideration of inherited norms and } \\
\text { principles that we may take for granted. }\end{array}$ \\
Algorithmic decision making & $\begin{array}{l}\text { Discussing practical applications of algorithmic decisions, with its uses, } \\
\text { misuses and limitations. }\end{array}$ \\
Environmental costs and considerations & $\begin{array}{l}\text { Considerations of how computing can damage our environment, and } \\
\text { ways we can try to reduce this impact. }\end{array}$ \\
Privacy and surveillance & $\begin{array}{l}\text { The implications of technology for our privacy, and discussing where we } \\
\text { should find ethical boundaries in addition to existing legal guidelines. } \\
\text { Many organisations have produced reports or ethical guidelines for their } \\
\text { particular contexts which are collected here. }\end{array}$ \\
Research culture & $\begin{array}{l}\text { Pieces related to the settings we do research in including pieces on team } \\
\text { science, open science, white supremacy and colonialism. } \\
\text { Examples of places where data ethics has been 'played out' in the real } \\
\text { world, or theories and frameworks on how to practically implement good } \\
\text { ethical practices. }\end{array}$ \\
Field specific & $\begin{array}{l}\text { Sub-sections around Natural Language Processing, Computer Vision, } \\
\text { and Explainable AI/ML which contain pieces specific to these fields; } \\
\text { these tend to be more technically focused. }\end{array}$ \\
\hline
\end{tabular}

Table 1. A list of the sub-sections of the reading list, with descriptions of the content included in each.

prompts. Secondly, the repository has integrated the All Contributors specification [17], which is a tool that allows us to record everyone who contributes to the repository, even if their contribution is not something that GitHub would typically recognise. To illustrate, the online repository currently has 30 contributors, but only eight of these are recognised by GitHub as those who have edited the repository itself. Other contributions we consider include attending meetings, emailing us with suggestions, making artwork for the repository, and generating ideas. Lastly the contents of the repository also generate a website ${ }^{4}$, that is served directly by GitHub pages. The website is rendered by Sphinx [18] and is re-built and deployed every time a new commit is added to the repository using Github Actions. This makes it very simple to keep the website up to date, even for those who are unfamiliar with front-end web development, since all of the website documentation is based on MyST flavoured markdown [19]. By hosting the materials openly on GitHub we appeal to data scientists and enable them to contribute easily; by mirroring these materials on a public facing website they become more accessible to those who are not familiar with GitHub.

\section{Data Ethics Club write-ups}

At each meeting we encourage attendees to write down salient points from their discussions in an open online document; these notes are then written up by one of the organisers and made into a summary of the discussion. There are currently 17 online. Including many people's contributions in the write-ups ensures that we can capture the range of thoughts and ideas expressed by different people over the course of the meeting. The summaries record the themes of our discussions and the diversity of perspectives that these inspire, as well as suggestions from attendees for further reading. We also use these summaries to share our thoughts and feedback with those who wrote or produced the content we discuss.

\section{DISCUSSION}

At the time of writing Data Ethics Club has been running for over a year, and in this time we have had been having regular fortnightly meetings and continually adding to the repository. We have benefited from a very wide range of people being part of our community, from those who work in different sectors, to those living in different countries (from the UK where the organisers are based to Peru, Australia, the USA, India and Italy to name a few), to experts in data ethics, deep learning and software engineering coming together. The Data Ethics Repository is shared with a CC-BY licence in the hope that others can re-use it and benefit from the collaborative effort that has gone into creating it. In fact, we have already been able to use the materials documented above to help other interested organisers set up their own data ethics discussion groups. We also hope others will be inspired to contribute their own ideas to the

${ }^{4}$ https: // dataethicsclub.com 
Data Ethics Club is a collaborative online project that warmly invites new people to join the community and use its resources. Ways you can get involved are:

- Suggest new content for the reading list (via email or GitHub).

- Attend, lead or write up a meeting.

- Create or review Pull Requests in order to, for example, update the website for the next meeting, put more suggestions on the reading list, or ensure all contributors are credited.

- Use the Data Ethics Club resources to run your own discussion group, or to create a reading list of your own or for students.

- Tell us know how you used the resources, or give us feedback on improving them.

repository and help us to improve it (see Box 1). In the following sections we will briefly discuss some of what we have learned in setting up and leading this project in the hope that our reflections will also be useful to others, as well as outlining some of the current limitations of the project and planned future developments.

\section{Feedback}

Feedback from attendees of the group, gathered through a small survey as well as unsolicited emails and comments, has been extremely positive. Attendees particularly highlighted the value of having the opportunity to discuss data ethics with a diverse group of people. The type of diversity in question varies, from a range of subject specialisms, to workplace contexts, to the countries our attendees live in. As mentioned previously, we believe the real benefit in discussing ethics in a group environment is to see outside of our own perspectives, and having a wide variety of attendees is an effective way to achieve this.

The Data Ethics Club format has also given us a voice as a community of people with interests and expertise in this area which we can use to affect change. For instance, we have been able to send feedback based on our discussion write-ups to calls such as those from the UK Statistics Authority about their proposed data ethics landscape review. We have also had discussions about the power of having a community to give each other the confidence to push back against decisions that we believe may be harmful, have difficult conversations with colleagues, or to go against the status quo. If we want to change the culture of responsibility in data science and bring ethics to the forefront then we cannot underestimate the benefit of having the support of a community behind us.

\section{Collaborative ethos}

Following the example of other open source projects that we admire (for instance, see The Turing Way [20]), the repository, and the group meetings of Data Ethics Club try to take an an ethos of collaboration. This means that as much as possible we want to share opportunities to own and lead the project, give a variety of options for how to get involved and give thanks and credit for everyone's contributions. Here are some ways we have tried to achieve this:

- Making the reading list (and everything else) open source.

- Using the All Contributors specification to credit everybody, including those not on GitHub.

- Giving people the option to contribute by writing as well as speaking.

- Making it easy for people to suggest papers, or get involved in organising, by sharing contact information regularly and inviting contributions often, and through different routes (e.g. email, GitHub, Twitter).

- Providing different ways for people to get involved that require various levels of commitment.

\section{Tips for coordinating an online journal club}

In organising Data Ethics Club we have developed our processes for organising and running an online discussion group. The Data Ethics Club was established during the coronavirus pandemic, and so online was the default option at the time, however we have found this has actually had a huge benefit. It has made it easy to reach beyond our immediate institution, and even outside of the country; we tend to have people from at least three different countries at each meeting. Based on this, the following is some advice for organising a group online based on our experiences.

- Make use of timezone sensitive tools. We use Time and Date ${ }^{5}$ to share the time of our next meeting, which makes it easy for people in different time zones to work out if they can come along.

${ }^{5}$ https: //timeanddate.com/ 
- Make use of breakout rooms. We have found it works well to host a small-group discussion for the majority of the meeting, and then use the larger group setting to hear from each small group and gather general feedback. This enables everyone to participate fully in the conversation, while also hearing a wide range of views. This is evident in the wildly different paths that conversation takes in each breakout room. We suggest having 4-6 people in each small group.

- Establish and share the Code of Conduct $(\mathrm{CoC})$ with a reminder at each session. Other groups are welcome to reuse the one we have shared on our repository that was adapted from a mixture of in-person journal club CoCs, and those from open source communities.

- Make sure that the discussion content is not too long or complex. Often people do their best to read or view it before attending but not everyone will have done, and so something that does not present a barrier to getting involved and that can be quickly summarised at the beginning is advisable.

- Send regular reminders. We especially find that reminders early on the day of the meeting are useful to those who are trying to attend around busy schedules.

- Enable people to provide anonymous feedback on the discussion and rate the discussion material. We use polls in Zoom for this purpose.

\section{Limitations and future developments}

Whilst we aim for our materials and repository to be useful, representative and inclusive there are limitations to the repository that we will work on as the project matures and develops. Firstly, to edit or use the repository a certain level of familiarity with git and GitHub is needed to be able to submit an issue, a pull request, or to reproduce and edit the repository for one's own purposes. We encourage people to get in touch via email for support and also provide detailed contribution guidelines to reduce this barrier, but it may nonetheless prevent input from those with useful insights who are less familiar with the tools that we use to organise. Secondly, the repository and group is UK-centric, English speaking, and holds meetings at GMT-friendly hours. Inevitably, this restricts the attendance of those from areas that do not share work hours in our timezone or who are not English speaking. By making our materials open and well-documented, we hope to enable and encourage Data Ethics Club groups to develop in other countries and time zones.

Lastly, we are aware that those who to invest time in regularly attending and contributing to the Data Ethics Club community are likely to be those with an existing interest in improving data science culture. As such, our community is likely to be a self-selecting sample of much wider data science and data ethics communities. Similarly, the community is also most likely to be made up of those who have the time, space and access to take part. One of our future aims is to find more ways to bring in a variety of opinions, including those from the public or other fields, to further broaden our perspectives.

\section{CONCLUSIONS}

The Data Ethics Club repository, and its associated community, will continue to develop as time goes on. For data ethics to become embedded in our daily practices, we need to understand that each of us has a role in preventing harm from data science outputs, and realise that data ethics is a practice for all of us to engage in collaboratively. We hope that this repository and the Data Ethics Club will be a starting point for sharing data ethics with other data scientists, and giving those with expertise in data ethics a chance to share their knowledge with the people who put it into action.

\section{ACKNOWLEDGEMENTS}

The Data Ethics Club community extends well beyond those who are the authors of this article, and so our thanks go to everyone who has contributed to the project by coming to a meeting, contributing to the reading list, helping us to maintain the website or just giving feedback.

ND is supported by an MRC GW4BioMed Studentship in Data Science and AI (MR/N013794/1). Thanks also go to the Jean Golding Institute for their support of this project.

\section{AUTHOR CONTRIBUTIONS}

ND and NZ jointly created the Data Ethics Club, and were jointly responsible for writing the original draft. NZ set up the software used for generating the website from the repository and created the images. ND, NZ and HD are the current organisers of Data Ethics Club and were all responsible for Data Curation, and Project Administration. All authors are contributors to the contents of the Data Ethics Club repository. All authors were responsible for reviewing and editing. 


\section{DECLARATION OF INTERESTS}

The authors do not declare any conflicts of interest.

\section{REFERENCES}

1. National Transport Study: Table NTS0907 2016. https ://www.gov .uk/government/collections/ national-travel-survey-statistics.

2. Crawford, K. Time to regulate AI that interprets human emotions. Nature 592, 167-167 (2021).

3. Chan-Olmsted, S. M. A review of artificial intelligence adoptions in the media industry. International Journal on Media Management 21, 193-215 (2019).

4. Buolamwini, J. \& Gebru, T. Gender shades: Intersectional accuracy disparities in commercial gender classification in Conference on fairness, accountability and transparency (2018), 77-91.

5. Wolff, J. Fairness, respect, and the egalitarian ethos. Philosophy \& public affairs 27, 97-122 (1998).

6. Godlovitch, S. Forbidding Nasty Knowledge: On the Use of Ill-gotten Information. Journal of applied philosophy 14, 1-17 (1997).

7. Redden, J., Dencik, L. \& Warne, H. Datafied child welfare services: unpacking politics, economics and power. Policy Studies 41, 507-526 (2020).

8. Wylie, C. D. Who Should Do Data Ethics? Patterns 1, 100015 (2020).

9. Gorman, M. E. Trading zones and interactional expertise: Creating new kinds of collaboration (Mit Press, 2010).

10. Practice tool: Developing a community of practice in your organisation 2021.

11. Taddeo, M. \& Floridi, L. What is data ethics? Philos Trans Ser A, 1-5 (2016).

12. Birhane, A. Algorithmic injustice: a relational ethics approach. Patterns 2, 100205 (2021).

13. Linzer, M. The journal club and medical education: over one hundred years of unrecorded history. Postgraduate medical journal 63, 475-478 (1987).

14. Orben, A. A journal club to fix science. Nature 573, 465-466 (2019).

15. Harding, S. Whose science? Whose knowledge? (Cornell University Press, 2016).

16. Attribution 4.0 International (CC BY 4.0) Creative Commons, Nov. 25, 2013. https : / creat ivecommons . org/licenses/by/4.0/.

17. All Contributors https://github.com/all-contributors/all-contributors.

18. Brandl, G. Sphinx documentation. http://sphinx-doc.org/sphinx.pdf (2021).

19. Community, E. B. Jupyter Book version v0.10. Feb. 2020. https : / / doi . org/10 . 5281/ zenodo . 4539666.

20. Arnold, B. et al. The Turing Way: a handbook for reproducible data science. Zenodo (2019). 